\title{
COUNTING PROBLEMS IN MODULI SPACE
}

\author{
ALEX ESKIN
}

In this series of lectures, we describe some counting problems in moduli space and outline their connection to the dynamics of the $S L(2, \mathbb{R})$ action on moduli space. Much of this is presented in analogy with the space of lattices $S L(n, \mathbb{R}) / S L(n, \mathbb{Z})$.

\section{LECTURE 1: Counting PROBlems AND VOlumes of STRATA}

Recall that $\Omega_{n}=S L(n, \mathbb{R}) / S L(n, \mathbb{Z})$ is the space of covolume 1 lattices in $\mathbb{R}^{n}$. This space is non-compact, since we can have arbitrarily short vectors in a lattice.

We will refer to moduli spaces of translation surfaces as defined in the lectures by Howard Masur in this volume [Ma1, Definition 6] as strata. Note that the case of $n=2$ in the space of lattices and the case of the stratum $\mathcal{H}_{1}(\emptyset)$ boil down to the same thing, since we are considering the space of unit area holomoprphic 1-forms on tori, which is given by $S L(2, \mathbb{R}) / S L(2, \mathbb{Z})$.

Let $B(R)$ be the ball of radius $R$ centered at 0 in $\mathbb{R}^{n}$. For a given lattice $\Delta \in \Omega_{n}$. we would like to find out how many lattice points, that is, how many points of $\Delta$ are contained in $B(R)$.

It is immediately clear that for a fixed lattice $\Delta$, as $R \rightarrow \infty$,

$$
|\Delta \cap B(R)| \sim \operatorname{Vol}(B(R))=\operatorname{Vol}(B(1)) R^{n} .
$$

(i.e. the number of lattice points is asymptotic to the volume). However, this is not uniform in $\Delta$. A uniform upper bound can be given as follows:

Let $\mathbb{R}^{n}$ be endowed with a Euclidean structure. Given a subspace $L$ of $\mathbb{R}^{n}$, we say it is $\Delta$-rational if $L \cap \Delta$ is a lattice in $L$. We define $d(L)$ to be the volume of $L /(\Delta \cap L)$. We then define the function $\alpha$ by

$$
\alpha(\Delta)=\sup \frac{1}{d(L)}
$$

where the supremum is taken over all $\Delta$-rational subspaces $L$. We have the following result (see [Sch]): there is a constant $C$, depending only

These notes are based on lectures of the author at Luminy, in June 2003. Thanks are given to Moon Duchin and Jayadev Athreya for taking detailed notes, and to the latter for typing them up. 
on the dimension $n$ so that for all $\Delta \in \Omega_{n}$,

$$
|\Delta \cap B(1)|<C \alpha(\Delta) .
$$

This estimate follows from what is called "the geometry of numbers".

The analogous problem in moduli space is as follows: let $\mathcal{H}(\beta)$ be a stratum, i.e. a moduli space of translation surfaces (defined in [Ma1, Definition 6]), and let $S=(X, \omega) \in \mathcal{H}(\beta)$. Recall (see e.g. [Ma1, §1.1]) that the holonomy of a curve $\gamma$ on $S$ is given by

$$
\operatorname{hol}(\gamma)=\int_{\gamma} \omega
$$

Let

$$
V_{s c}(S)=\{\operatorname{hol}(\gamma): \gamma \text { is a saddle connection on } S\}
$$

so that $V_{s c}(S) \subset \mathbb{C} \simeq \mathbb{R}^{2}$ (saddle connections are defined in [Ma1, Definition 3]). Note that $V_{s c}(S)$ is a discrete subset of $\mathbb{R}^{2}$, but it is not, in general, a subgroup. We are interested in $\left|V_{s c}(S) \cap B(1)\right|$, i.e. the number of saddle connections of length at most 1 on $S$.

The result is as follows: Fix $\epsilon>0$. Then there is a constant $c=$ $c(\beta, \epsilon)$ such that for all $S \in \mathcal{H}(\beta)$ of area 1 ,

$$
\left|V_{s c}(S) \cap B(1)\right| \leq \frac{c}{\ell(S)^{1+\epsilon}},
$$

where $\ell(S)$ is the length of the shortest saddle connection on $S$.

The proof of this result (which can be found in [EM]) is more difficult that that of (2). It uses techniques developed by Margulis for the quantitative version of the Oppenheim conjecture (see Lecture 3), as well as induction on the genus.

The following construction and its analogues play a key role. For any function of compact support $f \in C_{c}\left(\mathbb{R}^{n}\right)$, let $\hat{f}(\Delta)=\sum_{v \in \Delta \backslash 0} f(v)$. Note that if $f=\chi_{B(1)}$, we get $\hat{f}(\Delta)=|\Delta \cap B(1)|$. We have the Siegel formula: For any $f \in C_{c}\left(\mathbb{R}^{n}\right)$,

$$
\frac{1}{\mu\left(\Omega_{n}\right)} \int_{\Omega_{n}} \hat{f}(\Delta) d \mu(\Delta)=\int_{\mathbb{R}^{n}} f d \lambda,
$$

where $\mu$ is Haar measure on $\Omega_{n}=S L(n, \mathbb{R}) / S L(n, \mathbb{Z})$, and $\lambda$ is Lebesgue measure on $\mathbb{R}^{n}$.

The generalization of this formula to moduli space was developed, so the legend goes, by Veech while he listened to Margulis lecture on the Oppenheim conjecture. For $f \in C_{c}\left(\mathbb{R}^{2}\right)$ we define the Siegel-Veech transform $\hat{f}(S)=\sum_{v \in V_{s c}(S)} f(v)$. Just as above, if $f=\chi_{B(1)}, \hat{f}$ counts the number of saddle connections of length $\leq 1$. 
Just as we had the Siegel formula for lattices, here we have the SiegelVeech formula: There is a constant $b(\beta)$ such that for any $f \in C_{c}\left(\mathbb{R}^{2}\right)$, we have

$$
\frac{1}{\mu\left(\mathcal{H}_{1}(\beta)\right)} \int_{\mathcal{H}_{1}(\beta)} \hat{f}(S) d \mu(S)=b(\beta) \int_{\mathbb{R}^{2}} f
$$

where $\mu$ is the natural $S L(2, \mathbb{R})$ invariant measure on $\mathcal{H}_{1}(\beta)$, where $\mathcal{H}_{1}(\beta) \subset \mathcal{H}(\beta)$ is the hypersurface of translation surfaces of area 1 (this measure is defined in [Ma1, §3], or in the next section).

Let us sketch the proof of this result (essentially from [Ve], also reproduced in $[\mathrm{EM}]$ ). The first step (which is by far the most technical) is to show that $\hat{f} \in L^{1}\left(\mathcal{H}_{1}(\beta)\right)$, so that the left hand side is finite. This can be deduced e.g. from (3). Having done this, we denote the quantity on the left hand side of $(5)$ by $\varphi(f)$.

Thus we have a linear functional $\varphi: C_{c}\left(\mathbb{R}^{2}\right) \rightarrow \mathbb{R}$, i.e. a measure. But it also has to be $S L(2, \mathbb{R})$ invariant. Only Lebesgue measue and $\delta_{0}$, the delta measure at 0 are $S L(2, \mathbb{R})$ invariant. Thus we have $\varphi(f)=$ $a f(0)+b \int_{\mathbb{R}^{2}} f$. It remains to show $a=0$. Consider the limit of indicator functions $f=\chi_{B(R)}$ as $R \rightarrow 0$. Both sides of the equation tend to 0 , so we have that $a=0$, and thus our result.

Returning to lattices, we can apply literally the same arguments to prove the Siegel formula (4). Note that nothing was special about dimension 2 in the above proof sketch. Thus, we have almost proved (4) as well. To be precise, we currently have:

$$
\frac{1}{\mu\left(\Omega_{n}\right)} \int_{\Omega_{n}} \hat{f}(\Delta) d \mu(\Delta)=b \int_{\mathbb{R}^{n}} f d \lambda,
$$

for some constant $b$. We need to show $b=1$. Here, we once again use $f=\chi_{B(R)}$, but this time consider $R \rightarrow \infty$. Recall that $\hat{f}(\Delta)=$ $|\Delta \cap B(R)| \sim \operatorname{Vol}(B(R))$, for $R \gg 0$. Thus, we get $b=1$, and the Siegel formula.

We should remark that for the space of lattices the proof of the Siegel formula indicated above is not the easiest available. In fact, it is possible to avoid proving apriori that $\hat{f} \in L^{1}\left(\Omega_{n}\right)$. See [Sie] or [Cas] or [Ter] for the details.

We now show how to use the Siegel formula to calculate the volumes of the spaces $\Omega_{n}$. We first prove a variant of the formula. Recall that $v \in \Delta$ is primitive if there is no integer $n$ so that $v / n \in \Delta$. The analogue of (1) for counting primitive vectors is

$$
\left|\Delta_{\text {prim }} \cap B(R)\right| \sim \frac{1}{\zeta(n)} \operatorname{Vol}(B(1)) R^{n},
$$


where $\zeta(n)$ is Riemann's zeta function. Now for $f \in C_{c}\left(\mathbb{R}^{n}\right)$, let

$$
\tilde{f}(\Delta)=\sum_{v \in \Delta}^{\prime} f(v)
$$

where the prime indicates that we are summing over primitive vectors only. Now the proof of the Siegel formula given above shows that

$$
\frac{1}{\mu\left(\Omega_{n}\right)} \int_{\Omega_{n}} \tilde{f}(\Delta) d \mu(\Delta)=\frac{1}{\zeta(n)} \int_{\mathbb{R}^{n}} f d \lambda .
$$

The rest of the argument is heuristic. Consider $f=\chi_{B(\epsilon)}$ for some small positive $\epsilon$. We have that $\tilde{f}(\Delta)=0$ unless $\Delta$ has a primitive vector of length less than $\epsilon$. Note that if $v$ is a primitive short vector, then so is $-v$. It turns out that we can, in the limit as $\epsilon \rightarrow 0$, ignore the contribution to the integral of the lattices which have more then two primitive short vectors; thus we may assume that $\tilde{f}(\Delta)=2$. Now, let $v$ any one of the two primitive short vectors in $\Delta$, and consider a basis for $\Delta$ containing $v$. We may subtract multiples of $v$ from the other elements of the basis, to make them as short as possible. After this "reduction" procedure is complete, we get a basis for $\Delta$ containing $v$ where all the other elements are almost orthogonal to $v$. Then these other basis elements form an arbitrary lattice of dimension $n-1$, i.e. an element of $\Omega_{n-1}$. Thus, the left hand side of (7) is approximately

$$
\frac{2}{\mu\left(\Omega_{n}\right)} \frac{1}{2} \operatorname{Vol}(B(\epsilon)) \mu\left(\Omega_{n-1}\right)
$$

where the factor of 2 came from the value of $\tilde{f}$, the factor of $\frac{1}{2} \operatorname{Vol}(B(\epsilon))$ came from the integral over $v \in \mathbb{R}^{n}$, and the factor $\mu\left(\Omega_{n-1}\right)$ came from the integral over the rest of the basis (and we assumed that $\tilde{f}$ is always either 0 or 1 ). The right hand side of (7) is exactly equal to $\frac{1}{\zeta(n)} \operatorname{Vol}(B(\epsilon))$.

Doing this more carefully, and taking into account the normalizations of the measures (to be defined in the next lecture), we get, after sending $\epsilon \rightarrow 0$

$$
\frac{1}{\zeta(n)}=\frac{n-1}{n} \frac{\mu\left(\Omega_{n-1}\right)}{\mu\left(\Omega_{n}\right)} .
$$

Now after iterating the above formula, we get the desired formula for the volume:

$$
\mu\left(\Omega_{n}\right)=\frac{1}{n} \zeta(2) \zeta(3) \ldots \zeta(n) .
$$

The above could be justified rigorously, but this is usually not done since (8) and (9) can be obtained from (7) in an easier way (see [Sie] 
or [Cas] or [Ter]). However, the analogue of the argument presented here is the only way we currently know how to proceed in the case of translation surfaces. This was done in [EMZ] where we obtained the following result, which corresponds to (8). For any stratum (i.e. moduli space of translation surfaces) $\mathcal{H}_{1}(\beta)$, the coefficient $b(\beta)$ involved in $(5)$ can be expressed in the following form:

$$
b(\beta)=\sum_{\alpha<\beta} c(\alpha, \beta) \frac{\mu\left(\mathcal{H}_{1}(\alpha)\right)}{\mu\left(\mathcal{H}_{1}(\beta)\right)},
$$

where the sum is over lower dimensional strata $\alpha$ (which lie at the "boundary" of $\left.\mathcal{H}_{1}(\beta)\right)$, and $c(\alpha, \beta)$ are explicitly known rational numbers.

We note that (10) fails as a method for calculating the volumes, since (unlike the lattice case) we do not have an independent formula for $b(\beta)$. In the second lecture we will show that the volumes can be computed in a different way; then (10) can be used to evaluate $b(\beta)$. Also, we will see in the third lecture that $b(\beta)$ is the answer to a certain natural counting problem. The numbers $b(\beta)$, called the SiegelVeech constants, appear in some other contexts as well, in particular in connection with the Lyapunov exponents of the geodesic flow.

\section{LECTURE 2: LATTICE POINTS AND BRANCHED COVERS}

In this lecture we describe briefly another strategy for calculating volumes of moduli spaces of translation surfaces, which also has a parallel for the space of lattices. Recall that we are considering the moduli spaces $\mathcal{H}(\beta)$ of translation surfaces with singularity structure $\beta=\left(\beta_{1}, \beta_{2}, \ldots \beta_{n}\right)$, where $\beta_{i} \in \mathbb{N}, \sum \beta_{i}=2 g-2$. Let the set of singularities be denoted by $\Sigma$. We have $|\Sigma|=n$, and we have the first relative homology group of $S$ relative to $\Sigma$ (with coefficients in $\mathbb{Z}$ ):

$$
H_{1}(S, \Sigma ; \mathbb{Z})=\mathbb{Z}^{2 g+n-1} \text {. }
$$

We can pick a basis for the relative homology by selecting $g$ a-cycles, $g b$-cycles (from absolute homology), and $n-1$ relative cycles, where a relative cycle is a path with starts at some point of $\Sigma$ and ends at a different point of $\Sigma$.

Fix a $\mathbb{Z}$-basis $\gamma_{1}, \gamma_{2}, \ldots \gamma_{k}$ of $H_{1}(S, \Sigma ; \mathbb{Z})$, where $k=2 g+n-1$. We recall the following fact (see $[\mathrm{K}]$ ):

Theorem 1. The map $(X, \omega) \rightarrow\left(\operatorname{hol}\left(\gamma_{1}\right), \ldots, \operatorname{hol}\left(\gamma_{k}\right)\right)$ from $\mathcal{H}(\beta) \rightarrow$ $\left(\mathbb{R}^{2}\right)^{k}$ is a local coordinate system.

By pulling back Lebesgue measure on $\left(\mathbb{R}^{2}\right)^{k}$, we obtain a normalized measure $\nu$ on $\mathcal{H}(\beta)$. (For more details on the above constuction, see 
$[$ Ma1, §3].) Now, we would like to define a measure on the hypersurface $\mathcal{H}_{1}(\beta)$.

This is similar to the lattice setting, where if we pick a basis $v_{1}, v_{2}, \ldots v_{n}$ for our lattice $\Delta \subset \mathbb{R}^{n}$, we get a matrix in $M_{n}(\mathbb{R})$ by letting $v_{i}$ be the $i$ th column. Note that since our lattice is unit volume, our matrix has determinant 1 . We have a natural (Lebesque) measure $\nu$ on $M_{n}(\mathbb{R})$. Consider the det $=1$ hypersurface $\Omega_{1}$ (i.e., $S L(n, \mathbb{R})$ ). We define a measure $\mu$ on this space as follows: let $E \subset \Omega_{1}$, and let $C_{1}(E)$ be the cone over $E$ (i.e. the union of all line segments which start at the origin and end at a point of $E)$. We define $\mu(E)=\nu\left(C_{1}(E)\right)$. This yields a finite measure since we are considering a fundamental domain under the $S L(n, \mathbb{Z})$-action. This is in fact the measure used in the previous section in the case of lattices.

Returning to the setting of translation surfaces, recall that the area of our surface $S=(X, \omega)$ is given by

$$
\operatorname{Area}(S)=\frac{1}{2 i} \int_{X} \omega \wedge \bar{\omega}=\frac{1}{2 i} \sum_{i=1}^{g} \int_{a_{i}} \bar{\omega} \int_{b_{i}} \omega-\int_{b_{i}} \bar{\omega} \int_{a_{i}} \omega
$$

where $a_{i}$ and $b_{i}$ are the $a$ - and $b$-cycles on $\mathrm{X}$ respectively.

This gives that the area is a quadratic form in the local coordinate system, i.e.,

$$
\operatorname{Area}(X, \omega)=Q\left(\operatorname{hol}\left(\gamma_{1}\right), \ldots, \operatorname{hol}\left(\gamma_{k}\right)\right) .
$$

However, it is a degenerate form, since it only depends on the absolute cycles $a_{i}$ and $b_{i}$. We can mimic the lattice picture now: we define $\mu(E)=\nu\left(C_{1}(E)\right)$ for any subset $E \subset \mathcal{H}_{1}(\beta)$. This is the measure used in the previous section for the case of translation surfaces.

In what follows, we should really work inside each local coordinate chart as in Theorem 1 and then sum over the charts at the end (see [EO, $\S 3.2])$. But to simplify the presentation, we pretend there is only one chart. Let $\mathcal{F} \subset \mathcal{H}_{1}(\beta)$ denote a fundamental domain (for the relation of equivalence of translation surfaces) with rectifiable boundary, so that each translation surface corresponds to a unique point in $\mathcal{F}$. Then,

$$
\mu\left(\mathcal{H}_{1}(\beta)\right)=\mu(\mathcal{F})=\nu\left(C_{1}(\mathcal{F})\right)
$$

We now make a cosmetic step. Let $C_{R}(\mathcal{F})$ denote the cone of $\mathcal{F}$ extended to the hypersurface of area $R$-surfaces. Clearly

$$
\mu\left(\mathcal{H}_{1}(\beta)\right)=\nu\left(C_{1}(\mathcal{F})\right)=\frac{\nu\left(C_{R}(\mathcal{F})\right)}{R^{k}} .
$$

We have the following fact:

$$
\left|C_{R}(\mathcal{F}) \cap\left(\mathbb{Z}^{2}\right)^{k}\right| \sim \nu\left(C_{R}(\mathcal{F})\right)
$$


as $R \rightarrow \infty$, i.e. the number of lattice points in a cone is asymptotic to the volume. Ususally this is used to estimate the number of lattice points, but here we use this in reverse and estimate the volume by the number of lattice points. Thus, we get that

$$
\mu\left(\mathcal{H}_{1}(\beta)\right)=\frac{\nu\left(C_{R}(\mathcal{F})\right)}{R^{k}} \sim \frac{\left|C_{R}(\mathcal{F}) \cap\left(\mathbb{Z}^{2}\right)^{k}\right|}{R^{k}},
$$

or, equivalently,

$$
\left|C_{R}(\mathcal{F}) \cap\left(\mathbb{Z}^{2}\right)^{k}\right| \sim \mu\left(\mathcal{H}_{1}(\beta)\right) R^{k} .
$$

The equation (11) is not useful unless we can find an interpretation of the points of $C_{R}(\mathcal{F}) \cap\left(\mathbb{Z}^{2}\right)^{k}$. This is given by the following:

Lemma 2. $S=(X, \omega) \in C_{R}(\mathcal{F}) \cap\left(\mathbb{Z}^{2}\right)^{k}$ if and only if $X$ is a holomorphic branched cover of the standard torus of degree $\leq R, \omega$ is the pullback of $d z$ under the covering map, and all singularities branch over the same point.

Proof: Since $S \in C_{R}(\mathcal{F})$, area $(S) \leq R$. By definition, $S \in\left(\mathbb{Z}^{2}\right)^{k}$ is equivalent to $\operatorname{hol}\left(\gamma_{1}\right), \ldots, \operatorname{hol}\left(\gamma_{k}\right) \in \mathbb{Z}^{2}$. Fix a non-singular point $z_{0}$ on $S$, and define $\pi: S \rightarrow T$, where $T$ is the standard torus, by $\pi(z)=\int_{z_{0}}^{z} \omega$. Since $\int_{\gamma} \omega \in \mathbb{Z}+i \mathbb{Z}$ for any closed curve or saddle connection $\gamma$, this is a well defined covering map with all singularities branching over the same point. Since the torus is unit volume, the area of $S$ is equal to the degree of the covering.

Let $N_{\beta}(d)$ denote the number ${ }^{1}$ of branched covers of $T$ of degree $d$ with branching type $\beta$. (Note that $N_{\beta}(d)$ is defined in purely combinatorial terms).

Combining Lemma 2 with (11), we obtain the following: as $R \rightarrow \infty$,

$$
\sum_{d=1}^{R} N_{\beta}(d) \sim \mu\left(\mathcal{H}_{1}(\beta)\right) R^{k} .
$$

(This relation was discovered by Kontsevich and Zorich, and independently by Masur and the author.) Thus, we can compute $\mu\left(\mathcal{H}_{1}(\beta)\right)$ if we can compute the asymptotics of the left-hand-side of (12). This is a purely combinatorial problem.

Suppose we are considering a degree $d$ cover of the torus. Consider the standard basis $a$ and $b$ of curves on the torus (when the torus is viewed as the unit square, the curves correspond to the sides of the square). They give rise to permutations of the sheets, that is, elements

\footnotetext{
${ }^{1}$ In order for Theorem 3 below to hold, we should, when defining $N_{\beta}(d)$, weigh each cover by the inverse of its automorphism group. However this does not affect the asymptotics and can be ignored here.
} 
of the symmetric group $S_{d}$. We will abuse notation by denoting these permutations also by $a$ and $b$. Singularity types of covers correspond to different conjugacy classes of the commutator $a b a^{-1} b^{-1}$. A simple zero is a transposition, a double zero a three cycle, a two simple zeroes is a product of two transpositions, etc. (So for example, if we are considering the stratum $\mathcal{H}(1,1)$, the commutator will be in the same conjugacy class as a product of two transpositions.) The number of pairs $(a, b) \in S_{d} \times S_{d}$ satisfying such a commutation relation can be expressed as a sum over the characters of the symmetric group $S_{d}$.

However, simply looking at the conjugacy class of the commutator permutation does not guarantee that the resulting surface is connected. We wish to count only the connected covers. However, the disconnected ones dominate the count. If one knows the number of disconnected covers exactly, one can compute the number of connected covers (by using inclusion/exclusion to subtract off all the possible ways a cover can disconnect). Unfortunately, as one does that, the first $n$ terms in the asymptotic formula cancel. Still, it is possible, using the exact formula for the number of disconnected covers in [BO], to carry out the computation (see $[\mathrm{EO}]$ ). The result, is a fairly messy but computable formula for the volume $\mu\left(\mathcal{H}_{1}(\beta)\right)$.

There are two consequences of the above computations worth mentioning:

Theorem 3. The generating function $F_{\beta}(q)=\sum_{d=0}^{\infty} N_{\beta}(d) q^{d}$ is a quasi-modular form, that is, it is a polynomial in the Eisenstein series $G_{k}(q), k=2,4,6$.

Theorem 4. $\pi^{-2 g} \mu\left(\mathcal{H}_{1}(\beta)\right) \in \mathbb{Q}$, where $g$ is the genus of any surface in $\mathcal{H}(\beta)$.

Both of the above theorems were conjectured by Kontsevich. Further work showed that they hold also for the connected components of strata, and that similar results hold for spaces of quadratic differentials. We remark that Theorem 4 implies that the Siegel-Veech constants are rational.

For the space of lattices, one can carry out the same construction. The main difference is that one ends up counting unbranched covers of the standard torus $T^{n}$, or what is equivalent, sublattices of the standard lattice $\mathbb{Z}^{n}$. By computing the number of sublattices of $\mathbb{Z}^{n}$ of index at most $R$, and sending $R \rightarrow \infty$, it is not difficult to reproduce (9). 


\section{LECTURE 3: The Oppenheim COnjeCture And Ratner's THEOREM}

3.1. Counting cylinders and saddle connections. Recall that $V_{s c}(S)=$ $\{h o l(\gamma): \gamma$ is a saddle connection on $S\}$ where $S=(X, \omega)$ is a translation surface. We also define the analogous set:

$V(S)=\{h o l(\gamma): \gamma$ is a closed geodesic on $S$ not passing through singularities $\}$.

Note that any such closed geodesic is part of a cylinder (see [Ma1, §3]), and all the closed geodesics in the cylinder have the same holonomy. Thus, $|V(S) \cap B(R)|$ is the number of cylinders on $S$ of length at most $R$.

Masur proved the following:

Theorem 5. For all translation surfaces $S$ in a compact set, there are constants $c_{1}$ and $c_{2}$ so that for $R \gg 1$

$$
c_{1} R^{2}<|V(S) \cap B(R)| \leq\left|V_{s c}(S) \cap B(R)\right|<c_{2} R^{2} .
$$

The upper bound is proved in [Ma2] and the lower bound is proved in [Ma3]. The proof of the lower bound depends on the proof of the upper bound. Another proof of both the upper and lower bounds with explicit constants was given by Vorobets in [Vo1] and [Vo2]. Also see [EM] for yet another proof of the upper bound, which is influenced by ideas of Margulis.

We also note that there is a dense set of directions with a closed trajectory and thus a cylinder.

The following theorem, gives asymptotic formulas for the number of saddle connections and cylinders of closed geodesics on a fixed surface. It was first proved in this form in [EM], but many of the ideas came from [Ve], where a slightly weaker version was proved.

Theorem 6. For a.e. $S \in \mathcal{H}_{1}(\beta)$, we have

$$
\left|V_{s c}(S) \cap B(R)\right| \sim \pi b(\beta) R^{2}
$$

where $V_{s c}(S)$ is the collection of vectors in $\mathbb{R}^{2}$ given by holonomy of saddle connections on $S$, and $b(\beta)$ is the Siegel-Veech constant from Lecture 1, (whose value is given by (10)).

Similarly, for cylinders of closed geodesics, we have that there is a constant $b_{1}(\beta)$ so that

$$
|V(S) \cap B(R)| \sim \pi b_{1}(\beta) R^{2}
$$

where $V(S)$ is the collection of vectors given by holonomy along (imprimitive) closed geodesics not passing through singularities, and $b_{1}(\beta)$ is the associated Siegel-Veech constant. 
The following exposition will be along the lines of [EM], which was heavily influenced by [Ve]. To simplify the notation, we only deal with the case of saddle connections. Define $g_{t}=\left(\begin{array}{cc}e^{t} & 0 \\ 0 & e^{-t}\end{array}\right)$ and $r_{\theta}=$ $\left(\begin{array}{cc}\cos \theta & \sin \theta \\ -\sin \theta & \cos \theta\end{array}\right)$. Let $f$ be the indicator function of the trapezoid defined by the points

$$
(1,1),(-1,1),(-1 / 2,1 / 2),(1 / 2,1 / 2) .
$$

Lemma 7. We have $\int_{0}^{2 \pi} f\left(g_{t} r_{\theta} v\right) d \theta \approx \begin{cases}2 e^{-2 t} & \text { if } e^{t} / 2 \leq\|v\| \leq e^{t}, \\ 0 & \text { otherwise. }\end{cases}$

Proof. Let $U$ denote the trapezoid. Note that

$$
f\left(g_{t} r_{\theta} v\right) \neq 0 \Leftrightarrow g_{t} r_{\theta} v \in U \Leftrightarrow r_{\theta} v \in g_{t}^{-1} U .
$$

The set $g_{t}^{-1} U$ is the shaded region in Figure 1. From (13) it is clear that the integral in Lemma 7 is equal to ( $2 \pi$ times) the fraction of the circle which lies inside the shaded region $g_{t}^{-1} U$. If $v$ is too long or too short (not drawn), then the circle would completely miss the shaded region, and the integral would be zero. If it does not miss, then $(2 \pi$ times) the fraction of the circle in the shaded region is approximately $2 e^{-2 t}$, independent of $\|v\|$.

We now prove Theorem 6. Summing our formula from Lemma 7 over all $v \in V_{s c}(S)$ and recalling the definition of the Siegel-Veech transform $\hat{f}(S)=\sum_{v \in V_{s c}(S)} f(v)$, we get

$$
\frac{1}{2} e^{2 t} \int_{0}^{2 \pi} \hat{f}\left(g_{t} r_{\theta} S\right) d \theta \approx\left|V_{s c}(S) \cap B\left(e^{t}\right)\right|-\left|V_{s c}(S) \cap B\left(e^{t} / 2\right)\right| .
$$

Writing $R=e^{t}$, we can rewrite this as

$$
\frac{1}{2} R^{2} \int_{0}^{2 \pi} \hat{f}\left(g_{t} r_{\theta} S\right) d \theta \approx\left|V_{s c}(S) \cap B(R)\right|-\left|V_{s c}(S) \cap B(R / 2)\right| .
$$

This equation is key to the counting problem, since the right hand side counts saddle connections in an annulus, and the left hand side is an integral over (part of) an $S L(2, \mathbb{R})$ orbit. (The fact that we only have approximate equality does not affect the leading order asymptotics.) Now we are supposed to use some sort of ergodic theory to analyze the behavoir of integral on the left-hand-side of (14) as $t \rightarrow \infty$ (or equivalently as $R \rightarrow \infty$ ). 


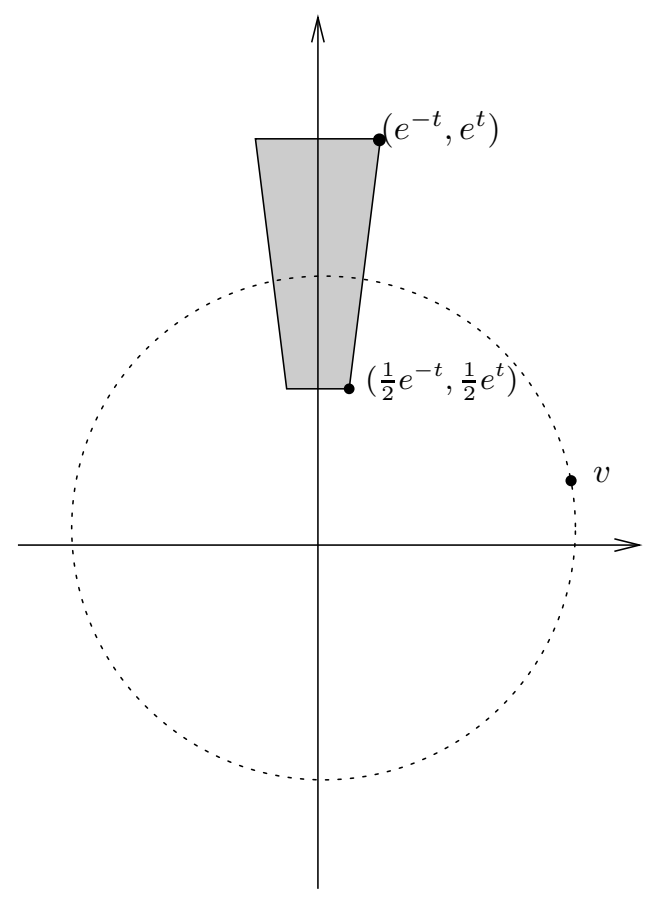

Figure 1. Lemma 7.

There is an ergodic theorem of Nevo [Ne] which implies that ${ }^{2}$ for almost all $S \in \mathcal{H}_{1}(\beta)$, and provided that $\hat{f} \in L^{1+\epsilon}\left(\mathcal{H}_{1}(\beta)\right)$, the integral converges to $2 \pi \int_{\mathcal{H}_{1}(\beta)} \hat{f}(S) d S=2 \pi b(\beta) \int_{\mathbb{R}^{2}} f$. The assertion that $\hat{f} \in$ $L^{1+\epsilon}$ can be verified using (3). This immediately implies Theorem 6 .

However, this approach is a failure if one wants to prove things about billiards in rational polygons: our theorems hold for almost every point $S$, and the set of translation surfaces arising from rational polygons has measure zero.

3.2. Oppenheim's conjecture. We now describe a counting problem for lattices which has a solution very similar to the above approach. (In fact, the results in this subsection predated and heavily influenced the discussion in the previous subsection). Let $Q=Q\left(x_{1}, x_{2}, \ldots x_{n}\right)$ be a indefinite irrational quadratic form in $n$ variables which is not a linear multiple of a rational form. In 1929 Oppenheim conjectured the following: $Q\left(\mathbb{Z}^{n}\right)$ is dense in $\mathbb{R}$. This was proved by Margulis in 1986 [Mar], using methods from dynamics and ergodic theory.

\footnotetext{
${ }^{2}$ The theorem of Nevo used here is about a general $S L(2, \mathbb{R})$ action, and uses nothing about the geometry of the moduli space.
} 
In $[\mathrm{EMM}]$, the following quantitative version of the conjecture is proved:

$$
\left|\left\{x \in \mathbb{Z}^{n}:\|x\| \leq T, a \leq Q(x) \leq b\right\}\right| \sim c(Q)(b-a) T^{n-2} .
$$

This is very similar to our above problem with saddle connections: we want to consider the lattice points in the ball of radius $T$ intersected with the region in between the two hypersurfaces $Q(x)=a$ and $Q(x)=$ $b$.

To solve this, one writes an integral very similar to the previous problem: this time, our compact group which we are integrating over is $H=S O(Q) \cap S O(n)$ and our diagaonal subgroup, denoted by $a_{t}$, has 1 's in every diagonal entry except the first and last, where they are $e^{t}$ and $e^{-t}$ respectively. Our integral is as follows: $T^{n-2} \int_{H} \hat{f}\left(a_{t} h \Delta_{Q}\right) d h$, where $\Delta_{Q}$ is a certain lattice in $\mathbb{R}^{n}$ associated to $Q$. We will assume that $Q$ has signature $(p, q)$, with $p \geq 3$ and $q \geq 1$.

Hence, if one makes a formal analogy between the spaces of translation surfaces and the spaces of lattices, the problem of counting saddle connections corresponds to the quantitative Oppenheim conjecture. There is an important difference between the two problems: unlike the saddle connection case where the result is "almost everywhere", we can prove the asymptotic formula (15) for ALL quadratic forms $Q$ not proportional to rational forms (and (15) fails for multiples of rational forms). This is due to the theorems we describe in the next part of the lecture, which are collectively known as Ratner's theorem. A major unsolved question is whether or not there is a version of Ratner's theorem for the action on the moduli space of translation surfaces. An affirmative answer would allow us to prove an asymptotic formula for billiards in every rational polygon (and every translation surface).

3.3. Ratner's theorem. Recall the Birkhoff Ergodic Theorem:

Theorem 8. Let $(X, \mu)$ be a measure space with $\mu(X)=1$, and let $T: X \rightarrow X$ be a ergodic measure preserving transformation. Let $f$ : $X \rightarrow \mathbb{R}$ be in $L^{1}(X, \mu)$. Then, for almost every $x \in X$, we have that

$$
\lim _{N \rightarrow \infty} \frac{1}{N} \sum_{i=0}^{N-1} f\left(T^{i} x\right)=\int_{X} f d \mu .
$$

This is a great theorem, but the "almost every" is fatal for most applications to number theory. We would like to know what happens for those other points as well, and Ratner's theorem can describe the behavior in certain settings.

First, however, recall that $T: X \rightarrow X$ is said to be uniquely ergodic if there is a unique invariant probability measure $\mu$ on $X$. 
We have the following consequence of unique ergodicity: if $T$ is uniquely ergodic, and $X$ is compact, then (assuming $f$ in continuous) the convergence in Birkhoff's theorem holds for all $x \in X$. To see this, let $\nu_{N}(f)=\frac{1}{N} \sum_{i=0}^{N-1} f\left(T^{i} x\right)$. Since $X$ is compact, the set of probability measures on $X$ is weak-* compact, so there is a subsequence $\nu_{n_{j}}$ and a probability measure $\nu_{\infty}$ so that $\nu_{n_{j}} \rightarrow \nu_{\infty}$. Its easy to see that $\nu_{\infty}$ is an invariant measure for $T$, so $\nu_{\infty}=\mu$. This is equivalent to (16).

Thus we can see that understanding the set of invariant measures is very important (or in particular, the set of ergodic invariant measures, since any invariant measure is a convex combination of ergodic measures). The other key issue in the topological setting is understanding the closure of orbits, and the two are related, since there will be invariant measures supported on orbit closures. This is the subject matter of Ratner's theorem (see [Rat1, Rat2, Rat3, Rat4, Rat5]).

We now describe the setting. Let $G$ be a semisimple Lie group with finite center (for example, $G=S L(n, \mathbb{R})$ ). Let $\Gamma$ be a lattice in $G$ (not neccesarily cocompact, e.g. $\Gamma=S L(n, \mathbb{Z})$ ), and let $U$ be a one parameter unipotent subgroup (for example, $u_{t}=\left(\begin{array}{ll}1 & t \\ 0 & 1\end{array}\right)$ ). We let $U$ act on $G / \Gamma$ by left multiplication on cosets (for $n=2$, this action is the horocycle flow).

The following theorem is stated somewhat informally. See e.g. [Rat5] for precise statements.

Theorem 9 (Ratner).

(1) The closure of every $U$-orbit is algebraic: that is, for all $x \in$ $G / \Gamma$, there is a closed subgroup $L \subset G$ such that $\overline{U x}=L x$, and that $L \cap x \Gamma x^{-1}$ is a lattice in $L$ (so that $L x$ is a closed subset of $G / \Gamma)$.

(2) Every ergodic $U$-invariant measure $\nu$ is algebraic, that is there exists a subgroup $L$ and $x \in G / \Gamma$, such that $\nu$ is the $L$-invariant measure on the closed subset $L x$.

(3) Every orbit is uniformly distributed in its closure, that is, for every $x \in G$ there exists a (not-nessesarily proper) subgroup $L$ of $G$ such that $L x=\overline{U x}$ is closed, and $\frac{1}{T} \int_{0}^{T} f\left(u_{t} x\right) d t \rightarrow$ $\int f(y) d \mu_{L}(y)$ as $t \rightarrow \infty$, where $\mu_{L}$ is the L-invariant probability measure on $L x$.

The second part of the theorem is the most difficult. The other two parts are essentially consequences of part 2. Also note that Birkhoff's theorem yields that for all $\epsilon>0$ there is a set $B$ of measure $<\epsilon$ so that outside of $B$, the convergence is uniform. Dani and Margulis 
obtained an explicit description of $B$ using part 2 of Ratner's theorem (see $[\mathrm{DM}]$ ).

One eventual goal is to prove a version of Ratner's theorem for the $S L(2, \mathbb{R})$ action on $\mathcal{H}_{1}(\beta)$. That is, we would like to classify invariant measures, orbit closures, and prove uniform distribution, for both the full $S L(2, \mathbb{R})$ action, and for the horocycle flow (which is defined to be the action on $\mathcal{H}_{1}(\beta)$ of the subgroup $\left(\begin{array}{ll}1 & * \\ 0 & 1\end{array}\right)$ of $\left.S L(2, \mathbb{R})\right)$.

One partial result in this direction is due to McMullen [Mc]: he has classified the $S L(2, \mathbb{R})$ orbit closures and invariant measures for the moduli space of genus 2 surfaces (i.e., the strata $\mathcal{H}(1,1)$ and $\mathcal{H}(2)$ ). Note that the integral in $(14)$ is over large circles in $S L(2, \mathbb{R})$, which can be approximated well by horocycles. Thus the horocycle flow is directly relevant to the counting problem. For other very partial results in this direction see [EMWM] and [EMS], where this program (i.e. measure classification with respect to the horocycle flow and application to counting) has been carried out in the very special case of branched covers of Veech surfaces.

\section{REFERENCES}

[BO] S. Bloch, A. Okounkov, The Character of the Infinite Wedge Representation, Adv. Math. 149 (2000), no. 1, 1-60

[Cas] J.W.S Cassels. An introduction to the geometry of numbers. Springer, 1959.

[DM] S.G. Dani and G.A. Margulis. Limit distributions of orbits of unipotent flows and values of quadratic forms. Advances in Soviet Math. 16(1993), 91-137.

[EM] A. Eskin and H. Masur, Asymptotic formulas on flat surfaces, Ergodic Th. and Dynam. Syst, v.21, 443-478, 2001.

[EMM] A. Eskin, G. Margulis, S. Mozes, Upper bounds and asymptotics in a quantitative version of the Oppenheim conjecture, Annals of Math., v. 147, 93-141, 1998.

[EMZ] A. Eskin, H. Masur and A. Zorich. Moduli Spaces of Abelian Differentials: The Principal Boundary, Counting Problems and the Siegel-Veech Constants. Publ. Math. Inst. Hautes Etudes Sci. No. 97, (2003), 61-179.

[EO] A. Eskin and A. Okounkov, Asymptotics of numers of branched covers of a torus and volumes of moduli spaces of holomorphic differentials, Invent. Math., 145 (1) (2001), 59-104.

[EMS] A. Eskin, H. Masur, M. Schmoll, Billiards in rectangles with barriers. Duke Math. J. 118 (2003), no. 3, 427-463.

[EMWM] A. Eskin, J. Marklof and D. Witte Morris, Unipotent flows and branched covers of Veech surfaces, In preparation.

[K] M. Kontsevich, Lyapunov Exponents and Hodge Theory, The mathematical beauty of physics (Saclay, 1996), 318-332, Adv. Ser. Math. Phys., 24, World Sci. Publishing, River Edge, NJ, 1997. 
[Mar] G.A. Margulis, Indefinite quadratic forms and unipotent flows on homgeneous spaces, in Dynamical Systems and Ergodic Theory, v. 23, 399-409, Banach Center Publ., PWN- Polish Scientific Publ., Warsaw, 1989.

[Ma1] H. Masur Ergodic theory of translation surfaces, Handbook.

[Ma2] H. Masur. The growth rate of trajectories of a quadratic differential., Ergodic Th. and Dynam. Syst, v.10, 151-176, 1990.

[Ma3]. H. Masur Lower bounds for the number of saddle connections and closed trajectories of a quadratic differential. In Holomorphic Functions and Moduli, Vol.1 D.Drasin ed. Springer-Verlag 1988, pp. 215-228

[Mc] C. McMullen. Dynamics of $S L_{2}(\mathbb{R})$ actions in genus 2. Preprint.

[Ne] A. Nevo, Equidistribution in measure preserving actions of semisimple groups, preprint.

[Rat1] M. Ratner. Strict measure rigidity for nilpotent subgroups of solvable groups. Invent. Math. 101 (1990), 449-482.

[Rat2] M. Ratner. On measure rigidity of unipotent subgroups of semisimple groups. Acta. Math. 165 (1990), 229-309.

[Rat3] M. Ratner. On Ragunathan's measure conjecture. Annals of Math. 134 (1991), 545-607.

[Rat4] M. Ratner. Raghunathan's topological conjecture and distributions of unipotent flows. Duke Math. J. 63 (1991), 235-290.

[Rat5] M. Ratner. Interactions between Lie groups, ergodic theory and number theory. Proc. of ICM (Zurich, 1994).

[Sch] W. Schmidt. Asymptotic formulae for point lattices of bounded determinant and subspaces of bounded height. Duke Math. J. 35(1968), 327-339.

[Sie] C. L. Siegel. Lectures on the geometry of numbers. Springer, 1989.

[Ter] A. Terras. Harmonic analysis on Symmetric spaces and Applications II Springer, 1988.

[Ve] W. Veech, Siegel measures. Annals of Mathematics 148 (1998), 895-944

[Vo1] Y. Vorobets. Ergodicity of billiards in polygons. Mat. Sb. 188 (3) (1997) pp. 65-112

[Vo2] Y. Vorobets. Periodic geodesics on translation surfaces. Eprint, arXiv:math.DS/0307249.

Department of Mathematics, University of Chicago, Chicago, IL 60637, USA.

E-mail address: eskin@math.uchicago.edu 